\title{
Five years and counting
}

\author{
Nature Photonics celebrated its fifth birthday in January this year. Now is a good time to recap what we \\ have done in the past, the current state of play and what we look forward to in the future.
}

Photonics is a unique field that involves both fundamental and applied research. A detailed knowledge of optical physics not only aids the study of the generation and detection of light, but also helps us understand how light manipulates and interacts with matter. Photonics is also an enabling technology that underpins a wide variety of important applications, including telecommunications, consumer electronics, lighting, displays, photovoltaics, materials processing, and medical imaging, diagnostics and surgery.

Since its launch in January 2007, Nature Photonics has constantly strived to publish top-quality exciting research on a platform that bridges both academia and industry across all the different disciplines of the field. With this objective in mind, we, as editors, endeavour to gather the best research articles and most exciting news from photonics laboratories around the world.

Our main focus is quality, not quantity this is our fundamental principle. In the early days, it was common for us to publish an average of 3-5 papers per month. Now, with a monthly paper count of around seven, we are witnessing a steady rise not only in the number of submissions but also in the quality of the research work submitted. Some believe that seven papers per month is a relatively low number. It is important to emphasize that we are in no way trying to restrict the number of papers we publish, but rather we are simply attempting to maintain the journal's high standard.

A common query from the community is whether we are more interested in publishing theoretical progress than technological breakthroughs. From what we have published so far ${ }^{1}$, it is self-evident that our focus is on both. Simply put, Nature Photonics provides its reader with research findings whose groundbreaking results, in terms of either novelty in conceptual understanding or significant advancement in technological performance, will be of immediate interest to the wider photonics community. Looking forwards, we will continue our strive to be the journal in which photonics researchers choose to disseminate their best and most inspiring research.

In tandem with the publication of primary research, we do from time to time organize Focus Issues dedicated to important and timely topics in photonics. So far we have

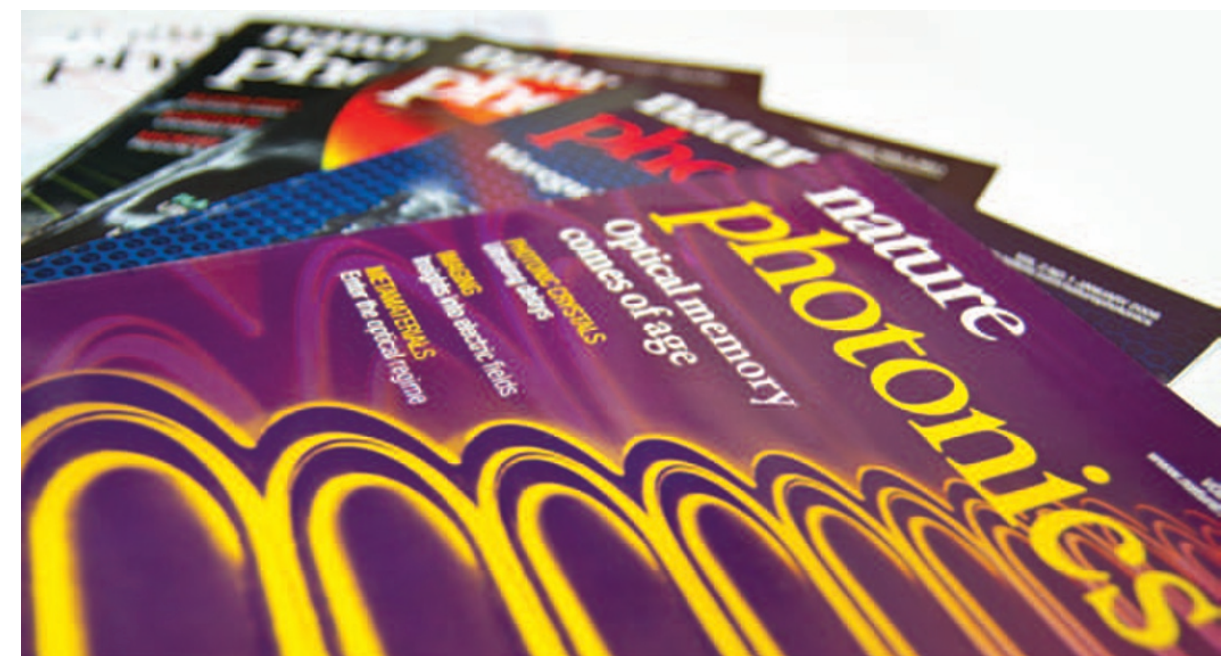

published eight Focus Issues ${ }^{2}$ covering the areas of slow light, super-resolution imaging, quantum optics, silicon photonics, $\mathrm{X}$-rays, next-generation metrology, optical manipulation and optofluidics. These issues generally consist of a collection of invited Review and Commentary articles, as well as an interview with an expert in the particular area. This year, there are three Focus Issues in the pipeline - the first being in March on the topic of photovoltaics. Do check it out.

Over the past five years, we have also published 26 issues of our Technology Focus supplement, which brings together articles and news regarding the emerging commercial applications of photonic technology ${ }^{3}$. Areas covered by the supplement include light-emitting diodes, semiconductor lasers, fibre lasers, semiconductor light sources, optical communications, displays, imaging, spectroscopy, optical data storage, photovoltaics, lithography, metrology, optical sensors, materials processing, adaptive optics, microwave photonics, ultrafast photonics, biophotonics, organic photonics and green photonics. Each supplement contains a collection of Industry Perspectives, Profile and Interview articles about industrial research and development, as well as research highlights, business news and product information. Although publication of Technology Focus ceased at the end of 2011, we will continue to report interesting and important photonic business news in the News and Views section of the journal.
Some of you may be surprised to learn that Nature Photonics also hosts its own conferences. In October 2007, we organized a technology conference on optical communications $s^{4}$. The unique three-day event brought together over 20 experts from academic institutions and industry to present their perspectives on the future evolution of optical network technology. In October 2010, we hosted another technology conference on the topic of photovoltaics ${ }^{5}-$ a timely subject, given the global call for a clean-energy economy. With 20 invited talks and two panel discussions, the three-day event provided a forum for discussing the current status and future outlook of the photovoltaic sector.

All in all, it has been a good five years. We have only been able to reach this milestone thanks to the diligence and efforts of our authors and reviewers, as well as the strong support we have received from our readers and the photonics community. We would like to extend our gratitude and appreciation to all of you for moulding the journal into the way it is today. We look forward to interacting more with you in the coming years and also to hearing any comments you may have, be they suggestions or criticisms. We wish you all the best for 2012 and beyond!

References

1. http://www.nature.com/nphoton/archive/subject.html

2. http://www.nature.com/nphoton/focus/index.html

3. http://www.nature.com/nphoton/archive/techfocus_index.html

4. http://www.nature.com/nphoton/supplements/ techconference2007/index.html

5. http://www.nature.com/nphoton/supplements/ techconference2010/pdf/photon_conf_report.pdf 